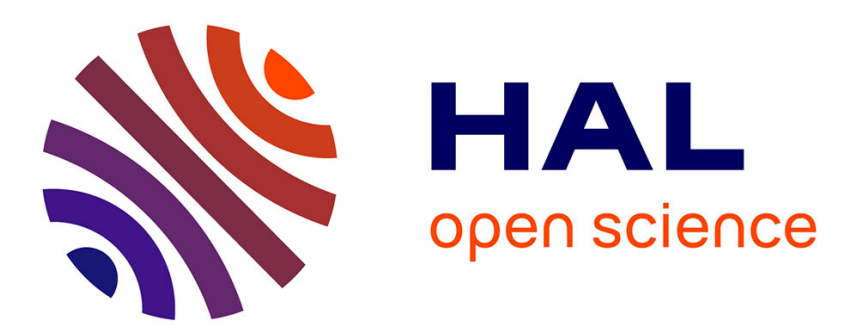

\title{
Two-Dimensional 1,3,5-Tris(4-carboxyphenyl)benzene Self-Assembly at the 1-Phenyloctane/Graphite Interface Revisited
}

\author{
Fabien Silly
}

\section{- To cite this version:}

Fabien Silly. Two-Dimensional 1,3,5-Tris(4-carboxyphenyl)benzene Self-Assembly at the 1Phenyloctane/Graphite Interface Revisited. Journal of Physical Chemistry C, 2012, 116, pp.10029. $10.1021 / j p 300678 \mathrm{~m}$. cea-00838043

\section{HAL Id: cea-00838043 https://hal-cea.archives-ouvertes.fr/cea-00838043}

Submitted on 24 Jun 2013

HAL is a multi-disciplinary open access archive for the deposit and dissemination of scientific research documents, whether they are published or not. The documents may come from teaching and research institutions in France or abroad, or from public or private research centers.
L'archive ouverte pluridisciplinaire $\mathbf{H A L}$, est destinée au dépôt et à la diffusion de documents scientifiques de niveau recherche, publiés ou non, émanant des établissements d'enseignement et de recherche français ou étrangers, des laboratoires publics ou privés. 


\title{
Two-dimensional
}

\section{1,3,5-Tris(4-carboxyphenyl)benzene self-assembly at the 1-phenyloctane/graphite interface revisited}

\author{
Fabien Silly*
}

CEA, IRAMIS, SPCSI, Hybrid Magnetic Nanoarchitectures, F-91191 Gif sur Yvette, France

E-mail: fabien.silly@cea.fr,tel:+33(0)169088019,fax:+33(0)169088446

*To whom correspondence should be addressed 


\begin{abstract}
This document is the unedited author's version of a Submitted Work that was subsequently accepted for publication in Journal of Physical Chemistry C, copyright (c) American Chemical Society after peer review. To access the final edited and published work see http://pubs.rsc.org/en/journals/journalissues/tc.
\end{abstract}

Two-dimensional (2D) self-assembly of star-shaped 1,3,5-Tris(4-carboxyphenyl)benzene molecules is investigated. Scanning tunneling microscopy reveals that this molecule can form three hydrogen-bonded networks at the 1-phenyloctane/graphite interface. One of these structures is close-packed and the two other ones are porous structures, with hexagonal and rectangular cavities. The network with rectangular cavities appears to be the most stable structure. 


\section{Introduction}

Engineering complex porous organic nanoarchitectures is the focus of recent research interest. ${ }^{1-4}$ Permanent nanoporosity can be exploited after subsequent functionalization ${ }^{5}$ for developing novel nanostructured materials dedicated to catalysis, gas storage, selective ion exchange, high density data storage, etc. Molecular self-assembly offers unique directions for the fabrication of two-dimensional (2D) organic nanoarchitectures. The resulting structures can be tailored at the nanometer scale by exploiting molecule-substrate ${ }^{6-8}$ or intermolecular interactions. ${ }^{1,3}$ Shape, size, structure and chemical composition of the molecular building blocks are key parameters driving the structure of self-assembled nanoarchitectures.

Strong intermolecular interactions are required to stabilize organic open networks and prevent the formation of close-packed structures. Exploiting the formation of hydrogen bonds (H-bonds) between neighboring molecular building blocks to create novel porous structures is particularly appealing because of the strength ${ }^{9}$ and the high selectivity and directionality of these bindings. ${ }^{10}$ Single ${ }^{11,12}$ and multicomponent ${ }^{1,13-16}$ self-assembled porous nanoarchitectures can be achieved through hydrogen bonding. Building blocks based on molecule having carboxyl groups $(\mathrm{COOH})$ are especially interesting because these groups can form $2 \mathrm{H}$-Bonds $(\mathrm{O}-\mathrm{H} \cdots \mathrm{O})$. Carboxyl group location in the molecule skeleton will have a huge impact on molecular self-assembly because it will guide the binding direction between neighboring molecules. ${ }^{17-21}$ For example triangular molecules having carboxyl group at the apex are expected to self-assemble into honeycomb structures. However Kampschulte et al. ${ }^{22}$ showed that 1,3,5-Tris(4-carboxyphenyl)benzene star-shaped molecules can form two distinc self-assembled structures at the liquid/graphite interface. They observed that the honeycomb network is the favored structure using aliphatic alcohol and fatty acid solvents, whose dielectric constant is lower or equal to 2.50. This was attributed to minimization of electrostatic effects. In comparison the influence of less polar solvents on molecular self-assembly appeared more ambiguous. Despite the low dielectric constant of alkane and arylalkane solvents, using these solvents did not lead to the same 1,3,5-Tris(4-carboxyphenyl)benzene orderings. An oblique and a honeycomb structure have been observed using dodecane solvent whereas the hon- 
eycomb structure was surprisingly the exclusive structure observed using 1-phenyloctane despite its low polarity. These observations did not allow determining which structure was most the stable or the lowest energy 2D nanoarchitecture.

In this paper we investigate the self-assembly of 1,3,5-Tris(4-carboxyphenyl)benzene molecules at the 1-phenyloctaone/graphite interface. 1-phenyloctane is a solvent having a low dielectric constant of 2.26 at $20.2^{\circ} \mathrm{C}^{23}$ Scanning tunneling microscopy (STM) reveals that the molecules can form three distinct two-dimensional hydrogen-bonded structures. Two of these structures are porous and one is close-packed. The open rectangular cavity structure appears to be the most stable structure.

\section{Experimental Section}

Solutions of 1,3,5-Tris(4-carboxyphenyl)benzene in 1-phenyloctane (98\%, Aldrich) were prepared. A droplet of this solution was then deposited on a graphite substrate. STM imaging of the samples was performed at the liquid/solid interface using a Pico-SPM (Molecular Imaging, Agilent Technology) scanning tunneling microscope. Cut Pt/Ir tips were used to obtain constant current images at room temperature with a bias voltage applied to the sample. STM images were processed and analyzed using the application FabViewer. ${ }^{24}$

\section{Results and Discussion}

The chemical structure of the 1,3,5-Tris(4-carboxyphenyl)benzene molecule is presented in 1 . This 3-fold symmetry molecule is a star-shaped molecule. Its skeleton is consisting in a central benzene ring connected to three peripheral 4'-benzoic acid groups. 1,3,5-Tris(4-carboxyphenyl)benzene molecules can form dimers, composed by two molecules rotated by $180^{\circ}$ with respect to each other as represented in 1 . These dimers are stabilized by two $\mathrm{O} \cdots \mathrm{H}-\mathrm{O}$ hydrogen bonds between neighboring molecules. 


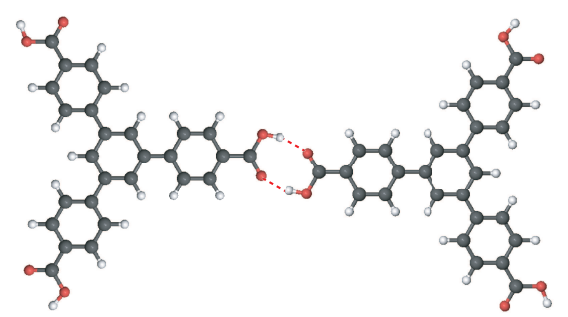

Figure 1: Scheme of hydrogen-bonded 1,3,5-Tris(4-carboxyphenyl)benzene $\left(\mathrm{C}_{27} \mathrm{H}_{18} \mathrm{O}_{6}\right)$ dimer. Carbon atoms are gray, oxygen atoms red, hydrogen atoms white, respectively. Red dotted lines represent molecular hydrogen bonds.

Large scale STM image reveals large 1,3,5-Tris(4-carboxyphenyl)benzene architectures at the 1-phenyloctane/graphite interface, 2a. High resolution STM images show that molecules selfassemble into a porous network. This structure is composed of rows of almost rectangular cavities, 2 b,c. The cavities are formed by two parallel molecular dimers. The doted line in $2 b$, perpendicular to the cavity rows, reveals that cavities of each odd (or even) rows are shifted with respect to another. Defects are locally observed in the molecular network, 2a,b; molecules are locally missing as highlighted by the dotted oval curve in $2 \mathrm{~b}$. Intramolecular features corresponding to the integrated density of states of the molecule appear distinctly in the high resolution STM images, 2b,c. The unit cell of the rectangular cavity network is represented by a blue dashed lines in $2 \mathrm{~d}$; it has a shape of a parallelogram with $3.1 \mathrm{~nm}$ and $1.7 \mathrm{~nm}$ sides and the acute angle of $\sim 75^{\circ}$ between them. Parallel molecular dimers (same molecular row) are connected through $\mathrm{O} \cdots \mathrm{H}-\mathrm{O}$ hydrogen bonds in this unit cell, whereas dimers of neighboring rows appear to be connected through $\mathrm{O} \cdots \mathrm{H}-\mathrm{C}$ hydrogen bonds.

The STM image presented 3 shows a second 1,3,5-Tris(4-carboxyphenyl)benzene self-assembled network . This network is locally observed on the graphite surface, less that $15 \%$ of the organic structures. Molecules form a honeycomb network with unit cell parameters of $3.1 \mathrm{~nm}$ and an angle of $\sim 60^{\circ}$. Individual molecules constituting the $2 \mathrm{D}$ struture are well-resolved in the STM images. The triangular molecules are located at the vertices of the hexagonal pores (3b. The model representing the honeycomb arrangement is shown $3 \mathrm{c}$. In this model the 1,3,5Tris(4-carboxyphenyl)benzene arrangement is stabilized by two $\mathrm{O} \cdots \mathrm{H}-\mathrm{O}$ hydrogen bonds between 


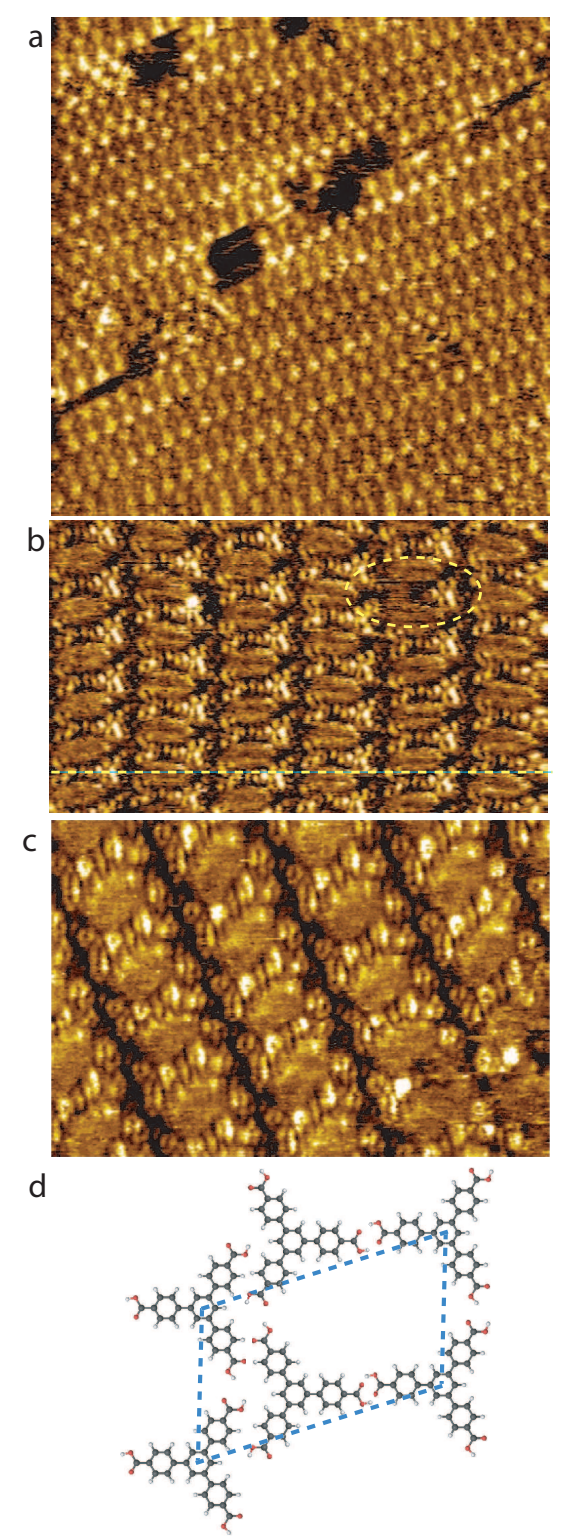

Figure 2: STM image of the 1,3,5-Tris(4-carboxyphenyl)benzene self-assembled porous network having rectangular cavities on graphite (a) $43 \times 43 \mathrm{~nm}^{2} ; \mathrm{V}_{s}=0.5 \mathrm{~V}, \mathrm{I}_{t}=18 \mathrm{pA}$, (b) $17 \times 10 \mathrm{~nm}^{2} ; \mathrm{V}_{s}$ $=0.4 \mathrm{~V}, \mathrm{I}_{t}=10 \mathrm{pA}$, (c) $14 \times 12 \mathrm{~nm}^{2} ; \mathrm{V}_{s}=-0.3 \mathrm{~V}, \mathrm{I}_{t}=30 \mathrm{pA}$. (d) Model of the network parallelogram unit cell (dashed blue lines). 


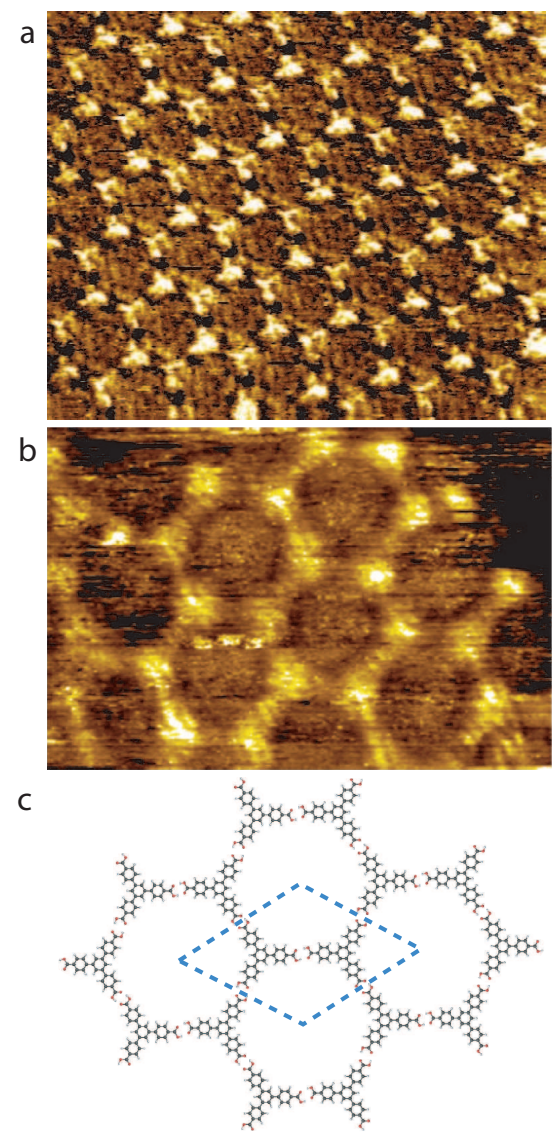

Figure 3: STM image of the 1,3,5-Tris(4-carboxyphenyl)benzene self-assembled honeycomb structure on graphite (a) $22 \times 19 \mathrm{~nm}^{2} ; \mathrm{V}_{s}=0.50 \mathrm{~V}, \mathrm{I}_{t}=15 \mathrm{pA}$, (b) $13 \times 9 \mathrm{~nm}^{2} ; \mathrm{V}_{s}=0.50 \mathrm{~V}$, $\mathrm{I}_{t}=18 \mathrm{pA}$. (c) Model of the hexagonal unit cell (dashed blue lines). 
molecular carboxylic groups.

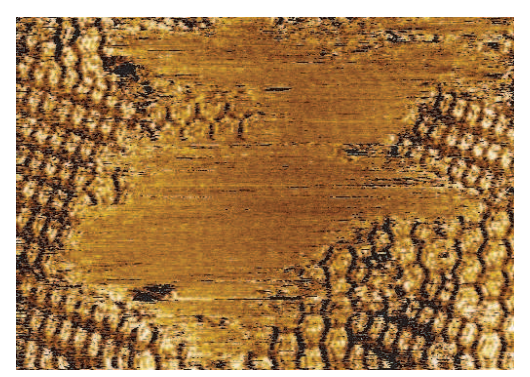

Figure 4: STM image of 1,3,5-Tris(4-carboxyphenyl)benzene network boundary, $22 \times 19 \mathrm{~nm}^{2}$; $\mathrm{V}_{s}=0.45 \mathrm{~V}, \mathrm{I}_{t}=10 \mathrm{pA}$.

4 shows an large scale image of 1,3,5-Tris(4-carboxyphenyl)benzene open network border. Due to STM tip modifications the STM image contrast is now reversed in comparison with previous STM images, i.e. molecules appear now dark and graphite surface appears bright. The STM image reveals that molecular honeycomb structure is mostly observed at the boundary of rectangular cavity network.

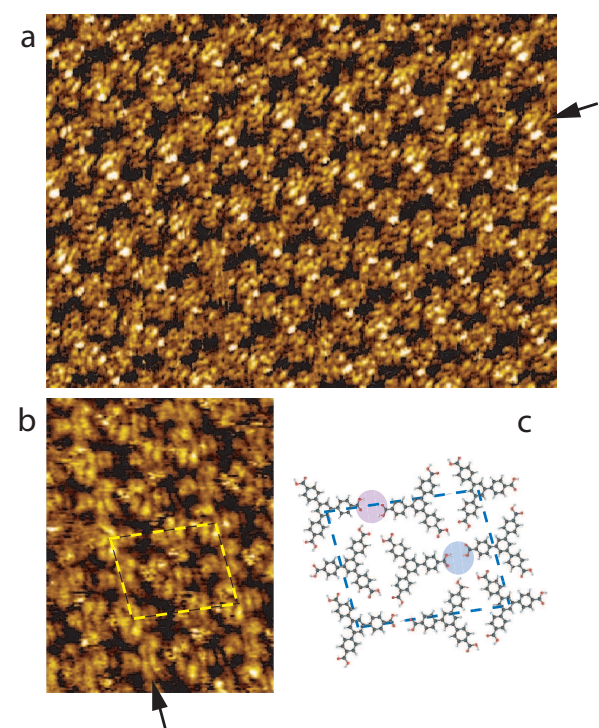

Figure 5: STM image of the 1,3,5-Tris(4-carboxyphenyl)benzene self-assembled close-packed structure on graphite (a) $19 \times 14 \mathrm{~nm}^{2} ; \mathrm{V}_{s}=0.50 \mathrm{~V}, \mathrm{I}_{t}=30 \mathrm{pA}$, (b) $6 \times 9 \mathrm{~nm}^{2} ; \mathrm{V}_{s}=0.50 \mathrm{~V}, \mathrm{I}_{t}=$ 20 pA. (c) Model of close-packed unit cell (dashed blue lines).

A third 1,3,5-Tris(4-carboxyphenyl)benzene 2D network is observed at the 1-phenyloctane/graphite interface, 5. The STM image shows that the molecules can also self-assemble into a close-packed structure. This structure represent less than $5 \%$ of the organic $2 \mathrm{D}$ architectures. The unit cell 
network is shown in 5 by a blue dashed line; it has a shape of a parallelogram with $3.1 \mathrm{~nm}$ and $2.4 \mathrm{~nm}$ sides and the acute angle of $\sim 83^{\circ}$ between them. This structure is composed of 1,3,5Tris(4-carboxyphenyl)benzene molecular rows. Along the molecular row (indicated by a black arrow in the STM images 5a,b) the molecules are rotated by $180^{\circ}$ with respect to their neighboring one. Neighboring molecular rows are connected through molecular carboxylic groups leading to the formation of 1,3,5-Tris(4-carboxyphenyl)benzene dimers. STM images show that the axis of dimer molecule is not aligned (as it is represented 1), i.e. neighboring molecular rows are shifted up or down depending of the dimer bonding. Down (up) binding is highlighted by purple (blue) ellipse in the model presented 5c, respectively. Similar row shift has been previously observed in the case of PTCDI-melamine network on $\mathrm{Au}(111)-(22 \times \sqrt{3}) .{ }^{14}$

In comparison with Ref., ${ }^{22}$ the STM images presented in 2, 3, 5 show that 1,3,5-Tris(4carboxyphenyl)benzene self-assemble into various two-dimensional nanoarchitures at the 1-phenyloctane/grap interface. Two of these networks are porous structures, 2 and 3 whereas one network is a closepacked structure, 5. All the observed networks are H-bonded structures. They share the same molecular building block, which is a 1,3,5-Tris(4-carboxyphenyl)benzene dimer stabilized by two hydrogen bonds as described in 1. Molecular dimers of the three observed networks are aligned in the same graphite crystallographic directions suggesting that molecule-substrate interaction is not favoring one structure over another. This suggests that molecule-molecule interaction is the key parameter driving 1,3,5-Tris(4-carboxyphenyl)benzene self-assembly. It should be noticed that rectangular cavity network dimers are placed by side leading the formation of a rectangular cavity whereas side dimers in the close-packed structure are shifted along the dimer axis by half dimer-length leading to the formation of a compact structure. Dimers are parallel in theses two 2D nanoarchitectures. In comparison dimers of the honeycomb structure are oriented at 120。 from each other. The three self-assembled structures were observed using different molecule concentration in the solution. Therefore molecular concentration in 1-phenyloctane does not appear modifying molecular self-assembly.

The honeycomb structure was previously observed in aliphatic alcohols and fatty acids sol- 
vent having a dielectric constant lower than 2.82 in Ref. ${ }^{22}$ This was attributed to minimization of electrostatic effect, i.e. more hydrophobic is the solvent, less polar and less dense are the generated self-assembled organic structures. However Ref., ${ }^{22}$ table 1, divulged that 1,3,5-Tris(4carboxyphenyl)benzene self-assemblies using 1-phenyloctane and dodecane solvents were deviating from predictions and were showing some discrepancy. Surprisingly the honeycomb structure was the only one observed at the 1-phenyloctane/graphite interface whereas the rectangular cavity and the honeycomb networks were both observed at the dodecane/graphite interface. These two solvents have very low dielectric constant (2.26 for 1-phenyloctane and 2.01 for dodecane) and they do not have any polar groups. One would not have expected to observe the honeycomb structure as the minimization of electrostatic effect using such solvent would have been quite ineffective in comparison with low dielectric constant fatty acids.

Our STM observations allow now elucidating the 1,3,5-Tris(4-carboxyphenyl)benzene selfassembly process at the 1-phenyloctane/graphite interface. Our STM images reveal that 1,3,5Tris(4-carboxyphenyl)benzene also self-assemble into the rectangular cavity network despite that 1-phenyloctane has a dielectric constant of 2.26. To be more precise STM images reveals that the open rectangular cavity network, which is denser than the honeycomb network, was not only the larger domain observed but also the structure that was dominantly observed. In comparison the honeycomb and close-packed networks were only locally observed. The honeycomb was especially observed at the rectangular cavity network boundary and was gradually disappearing with time passing. This suggests that the honeycomb structure gradually collapses to form the rectangular structure. After few hours we experimentally observed that the rectangular cavity network represents more than $80 \%$ of the organic self-assembled structures. In comparison the honeycomb structure and the close packed structure represent less than $15 \%$ and $5 \%$ respectively of the 1,3,5-Tris(4-carboxyphenyl)benzene nanostructures. Therefore these observations reveal that the rectangular cavity structure is the most stable structure at the 1-phenyloctane/graphite interface and the two other structures are metastable. Kitaigorodskii ${ }^{25}$ showed that close-packed structure are favored for weak interacting building blocks. As we observed open and close-packed structures 
that means that molecular structures results from the subtle balance between molecular interactions (hydrogen bonds) that may stabilize open structures and the tendency of molecule to form compact structures. Density functional theory (DFT) calculations would allow identifying the energetically most stable structure in the gas phase neglecting the influence of solvent and substrate on molecular self-assembly.

\section{Conclusion}

To summarize, scanning tunneling microscopy showed that 1,3,5-Tris(4-carboxyphenyl)benzene molecules self-assembled into three distinct networks at the 1-phenyloctane/graphite interface. The close-packed and the porous honeycomb networks were only locally observed suggesting that these structures are metastable. In comparison the porous rectangular cavity structure was the largest structure covering the surface. This suggests that this structure is the most stable nanoarchitecture using 1-phenyloctane solvent. Our results show that different molecular self-assembled structures can coexist and that exploring the controllability, predictability and stability of these structures at the nanometer scale is essential to elucidate self-assembly processes.

\section{Acknowledgement}

The research leading to these results has received funding from the European Research Council under the European Union's Seventh Framework Programme (FP7/2007-2013) / ERC grant agreement $\mathrm{n}^{\circ} 259297$.

\section{References}

(1) Liang, H.; Sun, W.; Jin, X.; Li, H.; Li, J.; Hu, X.; Teo, B. K.; Wu, K. Angew. Chem. Int. Ed. 2011, 50, 7562-7566. 
(2) Klyatskaya, S.; Klappenberger, F.; Schlickum, U.; Kühne, D.; Marschall, M.; Reichert, J.; Decker, R.; Krenner, W.; Zoppellaro, G.; Brune, H.; Barth, J. V.; Ruben, M. Adv. Funct. Mater. 2011, 21, 1230-1240.

(3) Yang, Y.; Wang, C. Chem. Soc. Rev. 2009, 38, 2576-2589.

(4) Stepanow, S.; Lin, N.; Payer, D.; Schlickum, U.; Klappenberger, F.; Zoppellaro, G.; Ruben, M.; Brune, H.; Barth, J. V.; Kern, K. Angew. Chem. Int. Ed. 2007, 46, 710-713.

(5) Silien, C.; Räisänen, M. T.; Buck, M. Small 2010, 6, 391-394.

(6) Deak, D. S.; Silly, F.; Porfyrakis, K.; Castell, M. R. Nanotechnology 2007, 18, 075301.

(7) Lu, C.; Zhu, E.; Liu, Y.; Liu, Z.; Lu, Y.; He, J.; Yu, D.; Tian, Y.; Xu, B. J. Phys. Chem. C 2010, 114, 3416-3421.

(8) Weckesser, J.; De Vita, A.; Barth, J. V.; Cai, C.; Kern, K. Phys. Rev. Lett. 2001, 87, 096101.

(9) Barth, J. V. Ann. Rev. Phys. Chem. 2007, 58, 375-407.

(10) Mura, M.; Silly, F.; Briggs, G. A. D.; Castell, M. R.; Kantorovich, L. N. J. Phys. Chem. C 2009, 113, 21840-21848.

(11) Baris, B.; Luzet, V.; Duverger, E.; Sonnet, P.; Palmino, F.; Cherioux, F. Angew. Chem. Int. Ed. 2011, 50, 4094-4098.

(12) Uemura, S.; Aono, M.; Komatsu, T.; Kunitake, M. Langmuir 2011, 27, 1336-1340.

(13) Gardener, J. A.; Shvarova, O. Y.; Briggs, G. A. D.; Castell, M. R. J. Phys. Chem. C 2010, $114,5859-5866$.

(14) Silly, F.; Shaw, A. Q.; Briggs, G. A. D.; Castell, M. R. Appl. Phys. Lett. 2008, 92, 023102.

(15) Palma, C.; Bjork, J.; Bonini, M.; Dyer, M. S.; Llanes-Pallas, A.; Bonifazi, D.; Persson, M.; ì, P. J. Am. Chem. Soc. 2009, 131, 13062-13071. 
(16) Sun, X.; Jonkman, H. T.; Silly, F. Nanotechnology 2010, 21, 165602.

(17) Zhou, H.; Dang, H.; Yi, J.; Nanci, A.; Rochefort, A.; Wuest, J. D. J. Am. Chem. Soc. 2007, $129,13774-13775$.

(18) Yokoyama, T.; Kamikado, T.; Yokoyama, S.; Mashiko, S. J. Chem. Phys. 2004, 121, 11993.

(19) Lackinger, M.; Heckl, W. M. Langmuir 2009, 25, 11307-11321.

(20) MacLeod, J. M.; Ivasenko, O.; Fu, C.; Taerum, T.; Rosei, F.; Perepichka, D. F. J. Am. Chem. Soc 2009, 131, 16844-16850.

(21) Ye, Y.; Sun, W.; Wang, Y.; Shao, X.; Xu, X.; Cheng, F.; Li, J.; Wu, K. J. Phys. Chem.C 2007, $111,10138-10141$.

(22) Kampschulte, L.; Lackinger, M.; Maier, A.; Kishore, R. S. K.; Griessl, S.; Schmittel, M.; Heckl, W. M. J. Phys. Chem. B 2006, 110, 10829-10836.

(23) Wohlfarth, C.; Wohlfarth, C.; Landolt, H.; BÃürnstein, R. Static dielectric constants of pure liquids and binary liquid mixtures; Springer, 1991.

(24) Silly, F. J. Microsc.-Oxford 2009, 236, 211-218.

(25) Kitaigorodskii, A. I. Acta Crystallographica 1965, 18, 585-590. 
Table of Contents (TOC) Image

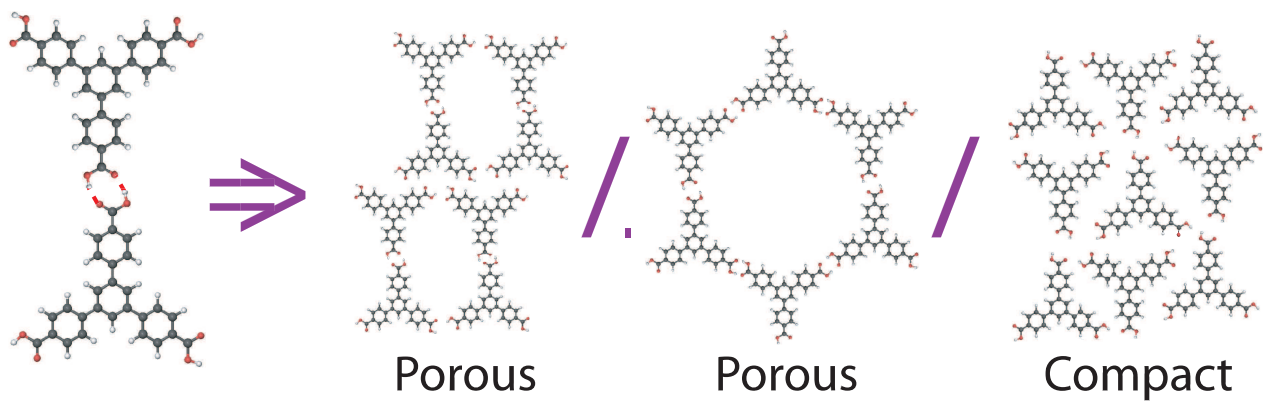

Figure 6: Table of Contents (TOC) Image 